\title{
Intermodal Raman Scattering between Orbital Angular Momentum Modes in Optical Fibers
}

Rottwitt, Karsten; Mariam Mathew, Neethu; Grüner-Nielsen, Lars; Rishøj, Lars Søgaard

Published in:

Proceedings of $22<$ sup $>$ nd $</$ sup $>$ International Conference on Transparent Optical Networks

Link to article, DOI:

10.1109/ICTON51198.2020.9203012

Publication date:

2020

Document Version

Peer reviewed version

Link back to DTU Orbit

Citation (APA):

Rottwitt, K., Mariam Mathew, N., Grüner-Nielsen, L., \& Rishøj, L. S. (2020). Intermgdal Raman Scattering between Orbital Angular Momentum Modes in Optical Fibers. In Proceedings of 22 International Conference on Transparent Optical Networks IEEE. https://doi.org/10.1109/ICTON51198.2020.9203012

\section{General rights}

Copyright and moral rights for the publications made accessible in the public portal are retained by the authors and/or other copyright owners and it is a condition of accessing publications that users recognise and abide by the legal requirements associated with these rights.

- Users may download and print one copy of any publication from the public portal for the purpose of private study or research.

- You may not further distribute the material or use it for any profit-making activity or commercial gain

- You may freely distribute the URL identifying the publication in the public portal 


\title{
Intermodal Raman Scattering between Orbital Angular Momentum Modes in Optical Fibers
}

\author{
Karsten Rottwitt, Neethu Mariam Mathew, Lars Grüner-Nielsen, and Lars Rishøj \\ Department of Photonics Engineering, DTU Fotonik, Technical University of Denmark, 2800 Kgs. Lyngby \\ Tel: (+45) 4525 6384, e-mail: karo@fotonik.dtu.dk
}

\begin{abstract}
This paper presents an experimental and theoretical analysis of Raman amplification of OAM modes i.e. beams carrying orbital angular momentum and spin angular momentum. We show that simple scalar equations are sufficient to describe the Raman interaction between OAM modes when working in a cyclic basis. The equations are similar to those used when considering Raman amplification in conventional fibers used for optical communication. Such fibers are typically single mode and support propagation of LP modes i.e. light that is linearly polarized. In fibers that support propagation of OAM modes as well as in fibers that support propagation of LP modes the scalar equations apply when considering very short- fiber lengths or long fiber lengths, only the proper gain efficiency needs to be used. Here, we discuss the gain efficiency, its material and modal dependencies and we demonstrate that the Raman gain efficiency among OAM modes in comparison with the efficiency among LP modes mainly differs due to a typically larger mode size of OAM modes relative to that of the fundamental LP modes in conventional fibers used for optical communication. In addition, we demonstrate a mode dependent loss in an optical fibers that support propagation of OAM modes.
\end{abstract}

Keywords: nonlinear optics, fiber optics, optical amplifiers, OAM modes.

\section{INTRODUCTION}

Raman amplifiers have attracted significant attention as a means to compensate for the attenuation in optical fibers, with application to high capacity optical communication. Until recently, research on Raman fiber amplifiers have focused on optical fibers that supports propagation of linearly polarized (LP) modes. This is because of several advantages; one is the capability of Raman amplifiers to provide distributed gain and hence improved noise performance as compared to discrete amplifications schemes [1]. Another advantage is that Raman amplifiers may provide gain at any signal wavelength assuming existence of a suitable pump. With the recent interest in using multiple spatial modes as a means to increase the capacity carried by a single optical fiber, an increasing interest in multimode optical fiber amplifiers have emerged. This has resulted in demonstration of amplifiers providing for example gain for 36 modes in an erbium doped fiber amplifier [2] or distributed Raman fiber amplification of orbital angular momentum modes [3]. Within optical communication, it has been suggested to use modes that carry spin- and orbital-angular momentum (OAM) rather than using LP modes. Using OAM modes, different communication channels may be carried by modes having different OAM. In view of Raman amplifiers, OAM modes are interesting since the intensity distribution of modes having different OAM may be nearly the same. Hence, since the Raman coupling among two modes only depends on the intensity overlap between the two interacting modes, it is expected that multimode OAM Raman amplifiers may be designed with a mode in-dependent gain, making them ideal candidates for multimode optical communications systems. The goal of this work is to describe and quantify the Raman gain efficiency of OAM modes. We investigate Raman gain in a multimode OAM fiber, and in particular the influence on the Raman gain efficiency due to the spin- and orbital-angular momentum as well as the influence due to the transverse intensity profile of the OAM modes and the intrinsic loss in state-of-the-art air-core OAM fibers.

\section{OAM MODES}

OAM modes are conveniently expressed in a cyclic basis defined by the basis vectors: $\hat{\sigma}^{ \pm}=(\hat{x} \pm \mathrm{i} \hat{y}) / \sqrt{2}$. In this basis the OAM modes are described by scalar radial field distributions: $f_{\ell m}$, where $\ell$ describes the topological charge also referred to as orbital angular momentum (OAM). In the electrical field of an OAM mode, the topological charge appears as a helical phase front where $\ell$ is the number of radial phase fronts within one cycle around the propagation axis. Index $m$ describes the radial mode number and is identical to the number of concentric intensity rings of the beam. $\hat{\sigma}^{ \pm}$describes the circular polarization of the mode and is referred to as the spin. Finally, an OAM mode is characterized by its handedness of the OAM and spin, having either the same handedness, spin-orbit aligned (SOa) modes, or opposite handedness, spin-orbit anti-aligned (SOaa) modes. That is [4]: SOa modes: $f_{l m}(r, \phi) \hat{\sigma}^{ \pm} e^{ \pm i l \phi} e^{i \beta_{\mu} z} e^{-i \omega t}$, and SOaa modes: $f_{l m}(r, \phi) \hat{\sigma}^{ \pm} e^{\mp i l \phi} e^{i \beta_{\mu} z} e^{-i \omega t}$, where $(r, \phi)$ are the transverse spatial coordinates, and $\exp ( \pm i \ell \phi)$ is the helical phase and $\beta_{\mu}$ is the propagation constant of mode $\mu$. For $\ell>1$ the SOa and SOaa modes occur in degenerate mode-pairs, respectively. However, for appropriate fiber designs the SOa and SOaa mode groups can be non-degenerate, thus enabling stable propagation without any mode coupling over few kilometers of fibers. It is noted that a major difference between 
LP modes and OAM modes is that $\hat{\sigma}^{ \pm}$for OAM modes is replaced by a unit vector having solely real components for LP modes. In the following, we investigate Raman interactions among OAM modes, seeded by the idea that in OAM modes information may be carried by the orbital angular momentum, while the intensity distribution of OAM mode of same radial order but different topological change is nearly identical.

\section{RESULTS AND DISCUSSION}

To design the simplest Raman amplifier consisting of a single pump and single signal at separate wavelengths, one need to solve two coupled equations. If the signal consists of multiple wavelength channels and further multiple spatial modes at each wavelength, the task involves solving multiple coupled equations. Furthermore, if multiple pump wavelengths and spatial pump modes are considered, then the number of coupled equations scales with the number of pump wavelengths and pump modes. In addition, the number of terms per equation that describe the transfer of energy from pump modes at various wavelengths to the signal modes increases dramatically with the number of pump wavelengths and pump modes. If furthermore, the signal and pumps consists of beams having complicated states of polarization, as for example OAM beams, the mathematical problem gets even more cumbersome since one needs to solve multiple coupled vectorial propagation equations involving two equations per signal mode and two per pump mode. However, in this work we show that the vectorial equations may be reduced to scalar equations when solving the equations in a cyclic basis. This holds for very short fibers where there is no coupling between the mode-pairs within the SOa mode-pairs nor within the SOaa mode-pairs. It also holds for long lengths of fibers. For sufficiently long lengths of fibers supporting LP modes, the state of polarization of the pump and signal goes through all states of polarization during propagation, consequently in such fibers the Raman gain coefficient is half of its maximum [5]. In analog for long lengths of fibers supporting OAM modes, the polarization state of the pump and signal also evolve into various polarization states described by the basis of SOa modes or SOaa modes, respectively [6] due to coupling between the mode-pairs in the SOa mode-pairs or the SOaa mode-pair, respectively. Consequently, the Raman gain efficiency in long length OAM fibers is also be averaged to half of its maximum [7]. The actual length needed for the scalar equations to be valid depends of the amplifier configuration. More specifically, when pump and signal propagate counter directional, the averaging happen over much shorter distances as compared to the case when the pump and signal are co-propagating. In the following, we consider co-propagating pump and signal.

In current state of the art single mode optical fibers used for optical communication, typical lengths of fiber Raman amplifiers are many kilometers long. Consequently, the gain due to the stimulated Raman process between one pump mode at one wavelength and one signal mode at another wavelength is conveniently described by two coupled scalar equations; one for the rate of change in signal power and one for the rate of change in pump power:

$$
\begin{gathered}
\frac{d P_{s}}{d z}=g_{r} P_{p} P s-\alpha_{s} P_{s} \\
\frac{d P_{p}}{d z}=-\frac{v_{p}}{v_{s}} g_{r} P_{p} P_{s}-\alpha_{p} P_{p},
\end{gathered}
$$

where $z$ is position, $g_{r}$ is the Raman gain coefficient, $P_{p}$ and $P_{s}$ are the pump and signal power levels, $\alpha_{p}$ and $\alpha_{s}$ are the attenuation rates at the pump and signal wavelengths, and $v_{p}$ and $v_{s}$ are the frequencies of the pump and signal. On the right hand side of eqn. (1a) the first term is the gain term, whereas the second term is the intrinsic fiber attenuation term. The first term on the right hand side of eqn. (1b) is the depletion term while the second term on the right hand side of eqn. (1b) is the intrinsic attenuation term for the pump.

When characterizing the gain coefficient, the signal power is kept as low as possible and the depletion term may then be ignored. In fact, ignoring the pump depletion is often a valid approximation, even when considering design of Raman amplifiers for optical communication systems. Under this assumption, the Raman gain, also denoted the on-off gain, is simply: $G_{R}=\exp \left(g_{r} P_{p} L_{e f f}\right)$, where $L_{e f f}=\left(1-\exp \left(-\alpha_{p} L\right)\right) / \alpha_{p}$ is the effective length of the amplifier fiber with physical length, $L$. It is noted that the signal gain, i.e. the signal output power relative to the signal input power, is the Raman gain times the intrinsic fiber loss: $\left[\exp \left(-\alpha_{s} L\right)\right]$.

The Raman gain coefficient depends on the material and the overlap between the pump and signal intensities. Mathematically, this is expressed through the Raman susceptibility tensor, $\chi^{R}$, weighted by the transverse intensity distributions of the pump and signal, multiplied by the normalized intensity overlap integral between pump and signal. Denoting the scalar field distributions of the signal and pump $f_{s}$ and $f_{p}$, respectively, where $f_{s}$ and $f_{p}$ are identified from $f_{l m}$, described in Section 2, the Raman gain coefficient is:

$$
g_{r}=\frac{\int \chi^{R}\left|f_{s}\right|^{2}\left|f_{p}\right|^{2} d a}{\int\left|f_{s}\right|^{2}\left|f_{p}\right|^{2} d a} \frac{\int\left|f_{s}\right|^{2}\left|f_{p}\right|^{2} d a}{\int\left|f_{s}\right|^{2} d a \int\left|f_{p}\right|^{2} d a},
$$

where the first factor of the product is the weighted overlap integral with the material and the second factor is the intensity overlap between the pump and signal modes. This is the inverse Raman effective area, that for $f_{s} \approx f_{p}$ equals the inverse effective area of the mode. When considering pump and signals in the fundamental $\mathrm{LP}_{01}$ 
mode, $g_{r}$ is for a standard single mode fiber typically around $1.4(\mathrm{~W} \cdot \mathrm{km})^{-1}$ when both the state of polarization of the pump and signal are linear and parallel to each other and remain so during the entire length of the amplifier.

When describing LP modes, the Raman susceptibility in standard Cartesian coordinates is used. In standard single mode fibers, i.e. pure silica fibers where the core region is weakly doped with germanium, the Raman susceptibility is to good approximation described by two quantities A and B provided in Table 1 [8]:

\begin{tabular}{c|c}
\hline$\chi_{i i i i}^{R}\left(-\omega_{s} ; \omega_{s}, \omega_{p},-\omega_{p}\right)=A+B$ & $\chi_{i j j i}^{R}\left(-\omega_{s} ; \omega_{s}, \omega_{p},-\omega_{p}\right)=B / 2$ \\
\hline$\chi_{i j i j}^{R}\left(-\omega_{s} ; \omega_{s}, \omega_{p},-\omega_{p}\right)=A$ & $\chi_{i i i j}^{R}\left(-\omega_{s} ; \omega_{s}, \omega_{p},-\omega_{p}\right)=B / 2$ \\
\hline
\end{tabular}

Table 1. In glass the Raman response may be described through the third order induced polarization $P_{i}^{(3)}=\varepsilon_{0} \chi_{i j k l}^{R} E_{j}^{S}\left(E_{k}^{p}\right)^{*}$, where index $i, j, k, l$ refer to the vectorial component and index $p, s$ refer to the pump and signal electric field

where $\mathrm{A}+\mathrm{B}$ is directly related to the Raman coupling among parallel-polarized beams, whereas $\mathrm{B} / 2$ is related to the Raman coupling among orthogonal polarized beams, e.g. an $x$-polarized signal and an $y$-polarized pump. In pure silica, A is much larger than B, especially at large frequency shift between the pump and signal, and often B is assumed negligible. When working in the cyclic basis, a scalar description of the Raman interaction, similar to the one described above between a pump and a signal in optical fibers that support propagation of LP modes may be used, however, only when the Raman susceptibility is transferred into the cyclic basis [9]. In the cyclic basis, the Raman susceptibility is shown in Table 2, where the quantities A and B are the same as in Table 1:

\begin{tabular}{c|c}
\hline$c_{i i i i}\left(-\omega_{s} ; \omega_{s}, \omega_{p},-\omega_{p}\right)=A+B / 2$ & $c_{i j j i}\left(-\omega_{s} ; \omega_{s}, \omega_{p},-\omega_{p}\right)=0$ \\
\hline$c_{i j i j}\left(-\omega_{s} ; \omega_{s}, \omega_{p},-\omega_{p}\right)=A+B / 2$ & $c_{i i j j}\left(-\omega_{s} ; \omega_{s}, \omega_{p},-\omega_{p}\right)=B$ \\
\hline
\end{tabular}

Table 2. Raman susceptibility $c_{i j k l}^{R}$ in cyclic coordinates $(i, j, j k, l) \in(R, L)$ where $(R, L)$ is associated with $\left(\hat{\sigma}^{-}, \hat{\sigma}^{+}\right)[7]$.

From Eqn. (2) and Table 1 and 2 the Raman amplification of a signal with a state of polarization that is parallel to that of the pump, e.g. having the same spin for OAM modes, but not necessarily the same topological charge, is nearly identical to what is expected when the pump and signal have the same linearly polarization. In [10] we have experimentally evaluated a Raman gain efficiency between a pump and signal with similar spin to be close to $0.7(\mathrm{~W} \cdot \mathrm{km})^{-1}$, i.e. reduced by a factor of two relative to the Raman gain efficiency among the fundamental $\mathrm{LP}_{01}$ modes in typical single mode high capacity transmission fibers.

In addition to the material, the Raman gain efficiency is also impacted by the overlap between the region of the most Raman active material, and the intensity of the pump and signal. In optical fibers, guiding is typically achieved by raising the refractive index of the guiding region relative to the surrounding material for example by doping silica with Ge. Since Ge doped silica has a significant higher Raman susceptibility compared to pure silica, one way to increase the Raman gain efficiency is to ensure that the overlap between the high refractive index region and the field is maximized, the first factor in eqn. (2). At best, the field is completely confined to the high index Ge doped region, in which case the weighted susceptibility simply equals the susceptibility of the material in the region of raised refractive index. Furthermore, to increase the Raman gain coefficient the Raman effective area needs to be minimized. However, since OAM modes have a phase singularity at the center of the beam, i.e. at $r=0$, they are constrained to intensity rings. Thus, even OAM modes at the lowest radial mode order, $m=1$, (one intensity ring) have a larger effective area than the fundamental $\mathrm{LP}_{01}$ mode in most telecommunication fibers. In [10] estimated to be around $160 \mu \mathrm{m}^{2}$, which is approximately a factor of two larger than the Raman effective area among two fundamental LP modes in typical transmission fibers.

The final part that influences the Raman gain efficiency is the effective length or in effect the attenuation rate of the pump light and the fiber length. Even though the attenuation of the signal also has a significant impact on the achieved output signal power as described above eqn. (2), we focus here only on the attenuation at the pump wavelength. In [10] we showed that the loss depend upon the topological charge of the OAM mode.

To analyze the attenuation in our fiber, we measured the loss of individual modes through the $1.2 \mathrm{~km}$ long fiber. We excited each OAM mode using a spatial light modulator. To measure the loss we used three broadband super-luminescent diodes. Together they covered a wavelength range from $1200 \mathrm{~nm}$ to $1700 \mathrm{~nm}$. The output light from the OAM fiber was focussed to a multimode fiber, which was coupled into an optical spectrum analyser used to measure the output spectrum. This was repeated for all the modes SOa and SOaa, for $\ell= \pm 5$, $\ell= \pm 6$, and $\ell= \pm 7$. The reference power was measured by cutting the fiber at $1.5 \mathrm{~m}$. The measured loss curves are shown in Fig. 1. During the measurements it was observed that the measured loss was the same for the SOa and SOaa modes, consequently, only one set of curves are shown The results obtained from the three sources are superimposed as the continuous lines. To confirm the reliability of the method, i.e. consistent coupling into the multimode pick-up fiber, the cutback measurement was repeated using multiple discrete wavelengths sources and by coupling the light directly from the OAM fiber into a free-space power meter. These measurements are included in Fig. 1 as markers and show good agreement with the before mentioned method.

The loss of the fiber has a strong and relative broad peak from $1350 \mathrm{~nm}$ to $1450 \mathrm{~nm}$. This wavelength range agrees with the $\mathrm{OH}$ absorption peaks that are present in silica fibers, typically denoted as 'water absorption'. The peaks at $1250 \mathrm{~nm}$ and $1390 \mathrm{~nm}$ corresponds to the $\mathrm{OH}$ absorption of $\mathrm{SiO}_{2}$, while the peak at $1420 \mathrm{~nm}$ 
corresponds to $\mathrm{OH}$ absorption of $\mathrm{GeO}_{2}$ [11]. It is noted, that the intensity of the peak at $1420 \mathrm{~nm}$ depends on the concentration of $\mathrm{GeO}_{2}$ doping in the fiber. In addition, it is interesting to note that the loss increases as $|\ell|$ decreases. We attribute this $\mathrm{OH}$ content at the inner boundary of the high index ring, since this is a silica-to-air interface, in combination with the fact that the lower order $|\ell|$ modes have higher intensity at this inner boundary. Thus, the high loss at the Raman pump wavelength can be attributed to a high $\mathrm{OH}$ content in the fiber, which may be reduced by further optimization of the fabrication process.

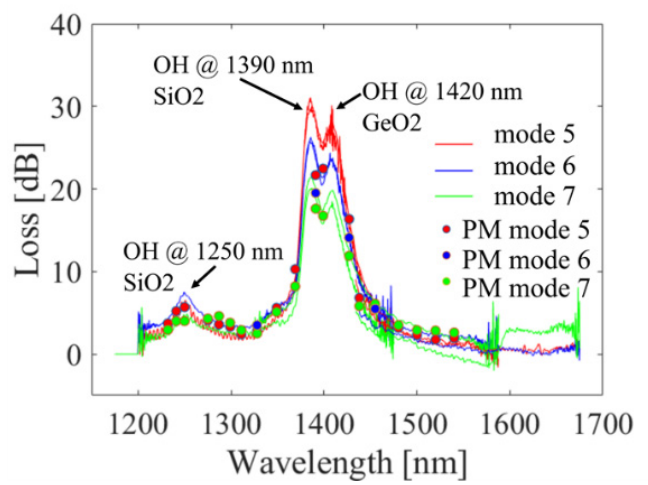

Figure 1. Measured loss versus wavelength in OAM fiber with a design as in [10]. Mode 5, 6 and 7 refer to SOa and SOaa mode groups with $|\ell|$ equal to 5, 6 and 7.

\section{CONCLUSIONs}

We have analyzed the Raman gain efficiency in an OAM amplifier. We have shown that simple scalar differential equations are sufficient to describe the Raman interaction between OAM modes. Finally, we have demonstrated a mode dependent loss in current state-of-the-art optical fibers.

\section{ACKNOWLEDGEMENTS}

P. Kristensen and OFS Fitel Denmark is thanked for fruitful discussions and for providing the fiber used for these measurements. The research was financially supported by the Danish National Research Foundation Centre of Excellence SPOC (DNRF213) and the Danish Council for independent research under Grant No. DFF 418400433.

\section{REFERENCES}

[1] T. N. Nielsen et al., "3.28-Tb/s transmission over $3 \times 100 \mathrm{~km}$ of nonzero-dispersion fiber using dual C- and L-band distributed Raman amplification," IEEE Photonics Technol. Lett., vol. 12, no. 8, p. 1079, 2000.

[2] Z. S. Eznaveh et al., "Ultra-low DMG multimode EDFA," in Proc. OFC 2017, paper Th4a.

[3] L. Zhu et al., "First demonstration of orbital angular momentum (OAM) distributed Raman amplifier over $18 \mathrm{~km}$ OAM fiber with data-carrying OAM multiplexing and wavelength-division multiplexing,", in Proc. OFC 2018, paper W4C.

[4] P. Gregg et al., "Conservation of orbital angular momentum in air-core optical fibers," Optica, vol. 2, no. 3, p. 267, 2015.

[5] Q. Lin et al., "Vector theory of stimulated Raman scattering and its application to fiber-based Raman amplifiers," J. Opt. Soc. Am. B, vol. 20, no. 8, p. 1616, 2003.

[6] G. Milione et al., "Higher order Pancharatnam-Berry phase and the angular momentum of light," Phys. Rev. Lett., vol. 108, p. 190401, 2012.

[7] K. Rottwitt et al., "Inter-modal Raman amplification of OAM fiber modes," APL Photonics, vol. 4, no. 3, 2019.

[8] R. Hellwarth, "Third order optical susceptibilities of liquids and solids," Prog. Quantum Electron., vol. 5, p. 1, 1977.

[9] K. Rottwitt et al., "Nonlinear Optics, Principles and Applications," CRC Press, 2014.

[10] K. Ingerslev, "Raman amplification of OAM modes," in Proc. CLEO Europe, 2017.

[11] A. Belov et al., "OH absorption in $\mathrm{GeO}_{2}$-doped fused silica fibres," Electron. Lett., vol. 18, p. 836, 1982. 